\title{
Tailoring Commercial Cellulose Membranes into Janus Conductive Electronic Skin via Diffusion- Controlled Polymerization
}

Feng Tang ${ }^{a} \perp$, Shenghong $\mathrm{Li}^{\mathbf{a}} \perp$, Hou-Yong Yu ${ }^{\text {a* }}$, Chuang Wang a ${ }^{\text {a }}$ Yingzhan $\mathrm{Li}^{\text {a }}{ }^{*}$, Ziheng Li ${ }^{\text {a }}$, Juming Yao ${ }^{\mathrm{a}}$, Jinhong Tang a and Jiaying Zhu ${ }^{\mathrm{a}}$.

a The Key Laboratory of Advanced Textile Materials and Manufacturing Technology of Ministry of Education, Zhejiang Sci-Tech University, Hangzhou 310018, China.

${ }^{\perp}$ F.T. and S. L. contributed equally to this work.

*Corresponding authors. Tel: +86-571-86843618; Fax: +86-571-86843619

E-mail addresses: phdyu@zstu.edu.cn (H.Yu) ;

yingzhanli@zstu.edu.cn (Y.Li) 
Figure S1 | The figure of the relationship between PANI thickness and ANI concentration s-3

Figure S2 | UV spectrum of CDM and CDM-PANI s-3

Table S1 | Comparison of various sensors on literature report s-4 


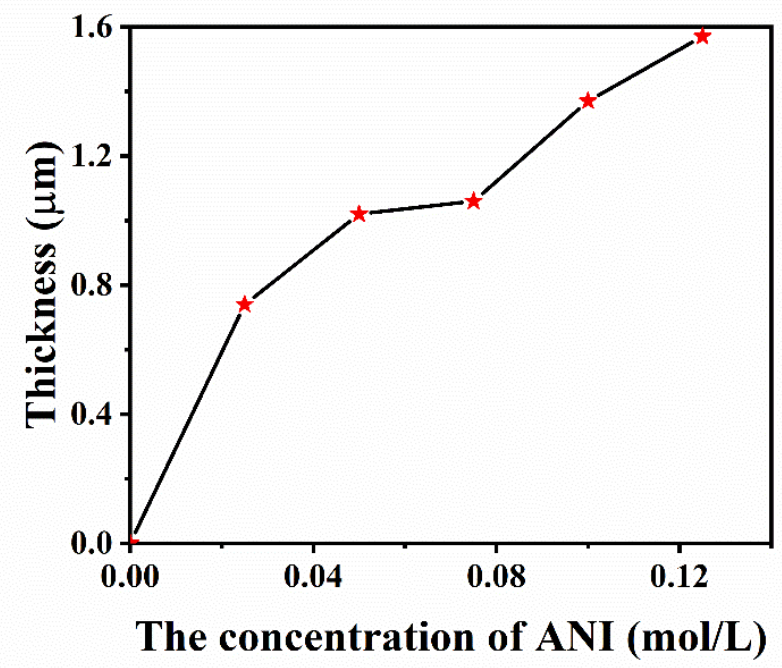

Figure S1. The figure of the relationship between PANI thickness and ANI concentration

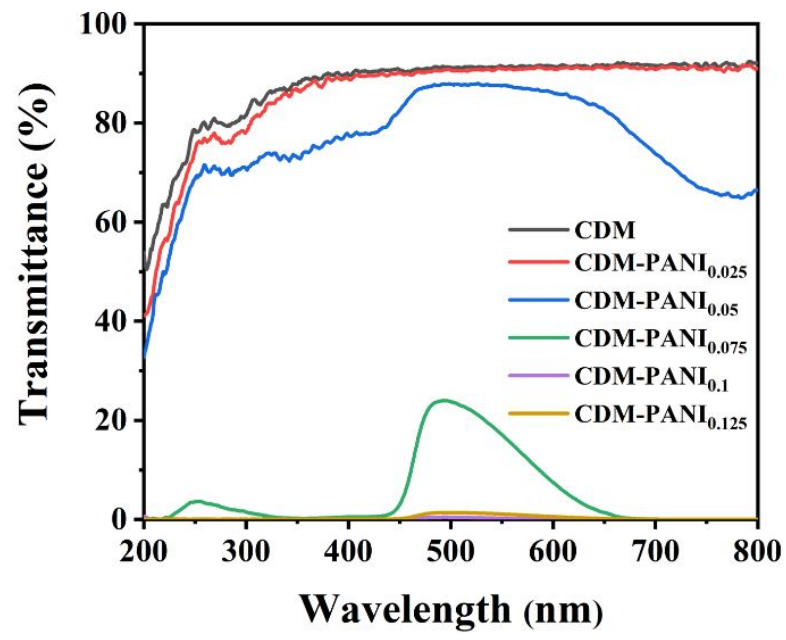

Figure S2. UV spectrum of CDM and CDM-PANI 
Table S1. Comparison of various sensors on literature report

\begin{tabular}{|c|c|c|c|c|}
\hline Sensing materials & Target gas & $\begin{array}{c}\text { Test } \\
\text { concentration } \\
(\text { ppm })\end{array}$ & $\begin{array}{l}\text { Response } \\
\left.\Delta R / \mathbf{R}_{\mathbf{0}}, \%\right)\end{array}$ & Ref. \\
\hline PVA/MWCNTs & \multirow{4}{*}{ Ethanol } & 100 & 0.56 & 1 \\
\hline $\mathrm{Pd} / \mathrm{SnO}_{2}-\mathrm{HSs}$ & & 50 & 0.90 & 2 \\
\hline MWCNTs-PEO & & 10 & 1.10 & 3 \\
\hline ZIF-67 & & 200 & 2.20 & 4 \\
\hline MWCNTs-PEO & \multirow{4}{*}{ Toluene } & 85 & 0.30 & 3 \\
\hline $\mathrm{SnO}_{2}$-microspheres & & 100 & 0.55 & 5 \\
\hline $\mathrm{Ni}_{\mathrm{x}} \mathrm{Co}_{1-\mathrm{x}} \mathrm{Fe}_{2} \mathrm{O}_{4}$ nanoparticles & & 100 & 0.60 & 6 \\
\hline $3 \mathrm{D} \mathrm{SiO}_{2}-\mathrm{RGO}$ & & 380 & 2.00 & 7 \\
\hline $\mathrm{Au}-\mathrm{In}_{2} \mathrm{O}_{3}$ & \multirow{4}{*}{ Formaldehyde } & 1 & 0.40 & 8 \\
\hline $\mathrm{rGO} / \mathrm{TiO}_{2}$ & & 1 & 0.65 & 9 \\
\hline $1 \mathrm{~mol} \% \mathrm{Pd}$-doped $\mathrm{SnO}_{2}$ & & 50 & 0.12 & 10 \\
\hline $\mathrm{CuO} / \mathrm{Pt}$ NPs & & 1 & 3.3 & 11 \\
\hline \multirow{3}{*}{ CDM-PANI 0.1} & Ethanol & & 1.90 & \multirow{3}{*}{$\begin{array}{l}\text { This } \\
\text { work }\end{array}$} \\
\hline & Toluene & 50 & 1.35 & \\
\hline & Formaldehyde & & 0.91 & \\
\hline
\end{tabular}




\section{Reference}

(1) Maity, D.; Rajavel, K.; Kumar, R. T. R. Polyvinyl Alcohol Wrapped Multiwall Carbon Nanotube (MWCNTs) Network on Fabrics for Wearable Room Temperature Ethanol Sensor. Sens. Actuators, B Chem. 2018, 261, 297-306. https://doi.org/10.1016/j.snb.2018.01.152.

(2) Xiao, L.; Xu, S.; Yu, G.; Liu, S. Efficient Hierarchical Mixed Pd/SnO2 Porous Architecture Deposited Microheater for Low Power Ethanol Gas Sensor. Sens. Actuators, B Chem. 2018, 255, 2002-2010. https://doi.org/10.1016/j.snb.2017.08.216.

(3) Zhou, Y.; Jiang, Y.; Xie, G.; Du, X.; Tai, H. Gas Sensors Based on Multiple-Walled Carbon Nanotubes-Polyethylene Oxide Films for Toluene Vapor Detection. Sens. Actuators, B Chem. 2014, 191, 24-30. https://doi.org/10.1016/j.snb.2013.09.079.

(4) Lü, Y.; Zhan, W.; He, Y.; Wang, Y.; Kong, X.; Kuang, Q.; Xie, Z.; Zheng, L. MOFTemplated Synthesis of Porous Co3O4 Concave Nanocubes with High Specific Surface Area and Their Gas Sensing Properties. ACS Appl. Mater. Interfaces 2014, 6 (6), 4186-4195. https://doi.org/10.1021/am405858v.

(5) Li, Y.; Chen, N.; Deng, D.; Xing, X.; Xiao, X.; Wang, Y. Formaldehyde Detection: SnO2 Microspheres for Formaldehyde Gas Sensor with High Sensitivity, Fast Response/Recovery and Good Selectivity. Sens. Actuators, B Chem. 2017, 238, 264-273. https://doi.org/10.1016/j.snb.2016.07.051.

(6) Msomi, J. Z.; Ndlovu, B.; Moyo, T.; Osman, N. S. E. Mössbauer and Magnetic Properties of Annealed NixCo1-XFe2O4 Nanoparticles. J. Alloys Compd. 2016, 683, 149-156. https://doi.org/10.1016/j.jallcom.2016.04.238. 
(7) Huang, D.; Li, X.; Wang, S.; He, G.; Jiang, W.; Hu, J.; Wang, Y.; Hu, N.; Zhang, Y.; Yang, Z. Three-Dimensional Chemically Reduced Graphene Oxide Templated by Silica Spheres for Ammonia Sensing. Sens. Actuators, B Chem. 2017, 252, 956-964. https://doi.org/10.1016/j.snb.2017.05.117.

(8) Im, D.; Kim, D.; Jeong, D.; Park, W. I.; Chun, M.; Park, J. S.; Kim, H.; Jung, H. Improved Formaldehyde Gas Sensing Properties of Well-Controlled Au Nanoparticle-Decorated In2O3 Nanofibers Integrated on Low Power MEMS Platform. J. Mater. Sci. Technol. 2020, 38, 5663. https://doi.org/10.1016/j.jmst.2019.09.002.

(9) Ye, Z.; Tai, H.; Xie, T.; Yuan, Z.; Liu, C.; Jiang, Y. Room Temperature Formaldehyde Sensor with Enhanced Performance Based on Reduced Graphene Oxide/Titanium Dioxide. Sens. Actuators, B Chem. 2016, 223, 149-156. https://doi.org/10.1016/j.snb.2015.09.102.

(10) Wang, J.; Zhang, P.; Qi, J. Q.; Yao, P. J. Silicon-Based Micro-Gas Sensors for Detecting Formaldehyde. Sens. Actuators, B Chem. 2009, 136 (2), 399-404. https://doi.org/10.1016/j.snb.2008.12.056.

(11) Lee, J. E.; Kim, D. Y.; Lee, H. K.; Park, H. J.; Ma, A.; Choi, S. Y.; Lee, D. S. Sonochemical Synthesis of HKUST-1-Based CuO Decorated with Pt Nanoparticles for Formaldehyde GasSensor Applications. Sens. Actuators, B Chem. 2019, 292 (April), 289-296. https://doi.org/10.1016/j.snb.2019.04.062. 